\title{
Die Auto Union AG und ihre Reaktionen auf das „Volkswagen"-Projekt 1931-1942
}

\author{
VON LUTZ SARTOR
}

\section{Überblick}

Es wird an einem Beispiel der Frage nachgegangen, wie sich die deutsche Automobilindustrie zum nationalsozialistischen Projekt eines Volkswagens ${ }^{1}$ und damit zu einem neuen Produkt auf dem umkämpften Kleinwagenmarkt verhalten hat. Die Auto Union AG eignet sich für diese Untersuchung besonders, ${ }^{2}$ da sie als zweitgrößter Kleinwagenproduzent Deutschlands in besonderem Maße von den Planungen zum Volkswagen betroffen war. Nach einleitenden Bemerkungen zu den Grundlagen des Volkswagen-Projekts und der Vorstellung des erfolgreichen Kleinwagens von DKW und später Auto Union wird untersucht, wie der Konzern auf den neuen Kleinwagen Volkswagen reagierte und seinen Spielraum ausschöpfte. Hierbei sind verschiedene Unternehmensstrategien und Grade der Aktivität zu verfolgen. Der Schwerpunkt lag auf Maßnahmen im Rahmen des Reichsverbandes der deutschen Automobilindustrie (RDA) und im Konzern selbst. Im Zusammenhang mit dem letzteren Punkt sind auch die im Auftrag der Firma angefertigten Berichte und Analysen eines externen Fachmannes von Interesse. Die Anstrengungen der Auto Union AG waren zwar alle als gescheitert anzusehen, behinderten aber in keiner Weise die weitere Entwicklung.

\footnotetext{
Abstract

This article presents an example of the way in which the German automobile industry responded to the national socialists' idea of a ,people's car"63 and, correspondingly, to a new product in the heavily contestet small car market.

1 Anstelle des ebenfalls gebräuchlichen Namens „KdF-Wagen“ wird durchgängig dieser Begriff verwendet.

2 Der 1998 bis 2000 im Sächsischen Staatsarchiv Chemnitz mustergültig erschlossene Bestand dieses Konzerns eröffnet die Möglichkeit, die Verhaltensweisen des Konzerns in den verschiedenen Stadien der Entwicklung des Volkswagen-Projektes nachzuzeichnen. Sächsisches Staatsarchiv Chemnitz: Findbuch zu den Beständen Auto Union AG, Horchwerke AG, Audi-Automobilwerke AG und Zschopauer Motorenwerke J.S. Rasmussen AG, bearbeitet von Martin Kukowski (= Veröffentlichungen der Sächsischen Archivverwaltung, Reihe A: Archivverzeichnisse, Editionen und Fachbeiträge, Bd. 1.1 und 1.2), Halle 2000.

3 The term „perople's car“ (,Volkswagen“) is used throughout this article in place of the other commonly used name „KdF-Wagen“.
} 
The Auto Union AG is particularly fitted for such a study, ${ }^{4}$ having been the second largest small car manufacturer in Germany and profoundly affected by the plan for the Volkswagen. Following a description of the founding of the Volkswagen project and a review on an earlier small car design of DKW and Auto Union, the author examines the company's reaction to the project und its testing of its limitations. Various corporate strategies and levels of activity are examined, as well as measures taken within the framework of the Reich Group of the German Automobile Industry (RDA) and within the company itself. Studies and reports on the latter produced by an outside specialist working under contract are also presented. The efforts undertaken by the Auto Union AG can be considered as a failure, without, however, hindering further develeopment.

\section{Zur historisch-politischen Entwicklung}

1.1 Die Motorisierung in Deutschland bis zum Anfang der 1930er Jahre

Der Gedanke, das Auto als Fortbewegungsmittel für breite Bevölkerungsschichten im Sinne einer „Volksmotorisierung“ zu gebrauchen, ist in Europa nicht erst durch die Nationalsozialisten seit Anfang 1933 vorgedacht und propagiert worden. Obwohl Kraftfahrzeuge bis nach dem Ersten Weltkrieg in Deutschland als Luxusobjekte und Statussymbole sehr vermögender Kreise galten, war schon kurz nach der Jahrhundertwende in der deutschen Fachpresse von der zukünftigen weiten Verbreitung des Automobils die Rede. ${ }^{5}$ Für die Zeit bis 1933 wird festgestellt, dass bis zu diesem Zeitpunkt „,unter dem Begriff, Volksautomobil' ${ }^{`}$ alle Versuche subsumiert wurden, größere Bevölkerungskreise zu motorisieren. ... Bereits ab 1904 werden an das ,Volksautomobil Forderungen nach Bequemlichkeit, leichter Handhabung, Gebrauchstüchtigkeit, preiswerter Anschaffung und Unterhaltung gestellt, die Henry Ford 4 Jahre später mit seinem ,Modell T" in Amerika verwirklichte" ${ }^{66}$ und in Deutschland

4 The written heritage of this corporation, exemplarily catalogued in the Saxon State Archives, Chemnitz, from 1998 to 2000, provides the opportunity to examine the behaviour of the firm at various stages of the Volkswagen project. Sächsisches Staatsarchiv Chemnitz: Findbuch zu den Beständen Auto Union AG, Horchwerke AG, Audi-Automobilwerke AG und Zschopauer Motorenwerke J.S. Rasmussen AG, bearbeitet von Martin Kukowski (=Veröffentlichungen der Sächsischen Archivverwaltung, Reihe A: Archivverzeichnisse, Editionen und Fachbeiträge, Bd. 1.1 und 1.2), Halle 2000.

5 Es ist in diesem Zusammenhang freilich nicht die Masse der Bevölkerung gemeint, sondern eine stetig, aber langsam wachsende Schicht von begüterten Bevölkerungsschichten, die sich die kostspielige Anschaffung und Unterhaltung eines Automobils leisten konnte.

6 Annette Gudjons: Die Entwicklung des „Volksautomobils“ von 1904 bis 1945 unter besonderer Berücksichtigung des „Volkswagens“. Ein Beitrag zu Problemen der Sozial-, Wirtschafts- und Technikgeschichte des Automobils, Hannover (Diss.) 1988, S. 48. Der Typ Ford T wurde von 1908-1927 mit über 15 Millionen Exemplaren hergestellt und begründete die herausragende Stellung der Firma. Zu Beginn der 1920er Jahre stammte fast die Hälfte der Welt-Kraftwagenproduktion von ihr. Reiner Flik: Von Ford lernen? Automobilbau und Motorisierung in Deutschland bis 1933, Köln, Weimar, Wien 2001, S. 5 u. 14. 
erst ein Vierteljahrhundert später die Nationalsozialisten populistisch aufgriffen. Der Zeitraum von 1918 bis 1933 wird in Deutschland als „Inkubationszeit" der Massenmotorisierung durch Personenkraftwagen angesehen. ${ }^{7}$ Es darf jedoch nicht übersehen werden, dass individuelle Mobilität in Deutschland von zwei anderen Verkehrsmitteln geprägt wurde: Vom Fahrrad mit einem Bestand von 20 Millionen Exemplaren $1939^{\circ}$ und, in weit geringerem Maße, vom Motorrad: Die deutsche Motorradproduktion lag in den Jahren 1925, 1926 und 1928 bis 1930 deutlich über der Automobilproduktion. ${ }^{9}$ Besonders dem Kleinkraftrad wird eine Pionierrolle bei der Vergrößerung der individuellen Motorradnutzung zugeschrieben. ${ }^{10}$ In den 1920er Jahren hatte Deutschland die höchste Motorraddichte der Welt, ${ }^{11}$ hier waren mehr Motorräder als Personenkraftwagen zugelassen. ${ }^{12}$ Die höchste Kraftwagendichte in Deutschland in den 1920er Jahren verzeichnete Mitteldeutschland. Die Reichsstatistik 1928 meldete darüber hinaus für Sachsen eine überdurchschnittliche PKW-Dichte. ${ }^{13}$

Ein wichtiger Ausgangspunkt für die Verstärkung der Debatte über einen Volkswagen bildete die ,Kleinwagenschwemme“ Anfang/Mitte der 1920er Jahre. ${ }^{14}$ Allein vom 1. Juli 1924 bis zum 1. Juli 1925 stieg die Zahl der PKW unter sechs ,Steuer-PS“15 um 60\% auf über ein Drittel aller PKW in Deutschland. ${ }^{16}$

7 Heidrun Edelmann: Vom Luxusgut zum Gebrauchsgegenstand. Die Geschichte der Verbreitung von Personenkraftwagen in Deutschland, (= Schriftenreihe des Verbandes der Automobilindustrie e.V. (VDA), Nr. 60), Frankfurt a.M. 1989, S. 16. Der Durchbruch zur „Massenmotorisierung“ wird für die alte Bundesrepublik erst für den Anfang der 1960er Jahre festgestellt, obwohl der PKW-Bestand in der BRD erstmals bereits 1952 das Vorkriegsniveau überstieg. Vgl. Dietmar Klenke: „Freier Stau für freie Bürger“. Die Geschichte der bundesdeutschen Verkehrspolitik 1949-1994, Darmstadt 1995, S. 17 u. 51.

8 Wolfgang König: Volkswagen, Volksempfänger, Volksgemeinschaft. „Volksprodukte“ im Dritten Reich. Vom Scheitern einer nationalsozialistischen Konsumgesellschaft, Paderborn 2004, S. 190.

9 Flik (wie Anm. 6), S. 292.

10 Edelmann (wie Anm. 7), S. 168.

11 Flik (wie Anm. 6), S. 12 u. 81.

12 Ebd., S. 80.

13 Ebd., S. 106 und Edelmann (wie Anm. 7), S. 92.

14 Allein von 1920 bis 1922 entstanden in Deutschland 67 Automobilwerke, von denen im Jahr 1926 nur noch 17 bestanden. Flik (wie Anm. 6), S. 307f. Nach einer anderen Quelle bestanden von 65 PKW-Produzenten, die 1924/25 existierten, 1928 noch 23 und 1930 nur noch 17. Edelmann (wie Anm. 7), S. 80. Eine positive Ausnahme in der Masse der meist nur kurzzeitig produzierten Kleinwagen war der Kleinwagen der Hannoverschen Maschinenbau AG (Hanomag), der wegen seiner charakteristischen Form unter dem Namen „Kommissbrot“ bekannt wurde. Von 1924 bis 1928 wurden von dem konkurrenzlos preiswerten Wagen über 15.000 Exemplare gebaut. Flik (wie Anm. 6), S. 155f.

15 Zur Berechnung siehe Angela Zatsch: Staatsmacht und Motorisierung am Morgen des Automobilzeitalters (= Schriften zur Rechts- und Sozialwissenschaft, Bd. 7), Konstanz 1993, S. 424, Anm. 2.

16 Die Zahl der PKW mit mehr als 14 „Steuer-PS“ stieg im gleichen Zeitraum nur minimal um zwei Prozent. Ebd., S. 418. 
Den ersten ausgereiften Kleinwagen mit Frontantrieb stellten die Zschopauer Motorenwerke J.S. Rasmussen AG unter dem Namen DKW Front F-1 1931 vor.

\subsection{Zur nationalsozialistischen Straßenverkehrspolitik ${ }^{17}$}

Nachdem die deutsche Reichsregierung unter Reichskanzler von Papen 1932 schon erste Steuererleichterungen für Kraftfahrzeuge eingeführt hatte, welche allerdings vor allem die gewerbliche Nutzung betrafen, setzte die nationalsozialistische Regierung diese Politik mit der Steuerbefreiung für neue Motorräder und PKW mit Verbrennungsmotor per 25. März 1933 fort. ${ }^{18}$ Allerdings war der Aufschwung der Automobilindustrie im ersten Halbjahr 1933 nicht darauf zurückzuführen, da er schon zuvor eingesetzt hatte. ${ }^{19} \mathrm{Ab}$ dem 31. Mai 1933 konnte zudem die Kraftfahrzeugsteuer für die künftige Nutzungsdauer einmalig abgelöst werden. ${ }^{20}$ Dies führte sowohl zu erheblichen Steuermehreinnahmen 1933 als auch zu einer Belebung des Gebrauchtund damit auch des Neuwagenmarktes..$^{21}$ Adolf Hitler kündigte schon bei der Eröffnung der Internationalen Automobil- und Motorradausstellung in Berlin am 11. Februar $1933^{22}$ die Förderung des Motorsports sowie ein umfangreiches Straßenbauprogramm an und wandte sich gegen die bisher praktizierte Unterordnung des Straßenverkehrs unter den Schienenverkehr. ${ }^{23}$ Die Wirkung dieser ,positiven Signale“ für die deutsche Kraftfahrzeugindustrie, die sich langsam im Frühjahr 1933 erholte, ${ }^{24}$ war eher psychologisch als direkt praktisch, aber dennoch nicht zu unterschätzen. ${ }^{25}$ Bereits am 1. Mai 1933 sprach Hitler von einem dem Automobilverkehr vorbehaltenen neuen Straßennetz. Dieses geplante Grundnetz von „Autobahnen“wuchs ständig: Wurden im August 1933 noch 7.000 Kilometer vorgesehen, so waren es nach

17 Vgl. Hans Mommsen u. Manfred Grieger: Das Volkswagenwerk und seine Arbeiter im Dritten Reich, Düsseldorf 1996.

18 Zur Kraftfahrwagensteuer in der Weimarer Republik siehe Angela Zatsch: Allen Fehlzündungen zum Trotz. Die Wirkung der Steuergesetzgebung auf die Verbreitung des Automobils (bis 1933), in: Harry Niemann u. Armin Hermann (Hg.): Die Entwicklung der Motorisierung im Deutschen Reich und den Nachfolgestaaten. Stuttgarter Tage zur Automobil- und Unternehmensgeschichte, Stuttgart 1995, S. 169-183 und dies. (wie Anm. 15), S. 411-506.

19 Christoph Buchheim: Die Erholung von der Weltwirtschaftskrise 1932/33 in Deutschland, in: Jahrbuch für Wirtschaftsgeschichte 2003, S. 13-26, hier S. $19 \mathrm{f}$.

20 Zatsch (wie Anm. 15), S. 489.

21 Ebd., S. 490f.

22 Es war die erste, die von einem deutschen Reichskanzler eröffnet wurde. Der Grund lag in der Erkrankung des eigentlich zur Eröffnung der Ausstellung vorgesehenen Reichspräsidenten Paul von Hindenburg.

23 Diese Schwerpunktsetzung soll eher zufällig geschehen sein. Edelmann (wie Anm. 7), S. 173.

24 Siehe z.B. Buchheim (wie Anm. 19), S. 19f.

25 Ian Kershaw: Hitler. 1889-1936, München 2002, S. 572. 
dem „Anschluss“ Österreichs bereits 12.000 und nach Kriegsbeginn über 20.000 Kilometer. Beim Baustop Ende 1941 betrug die Gesamtlänge der Autobahnen 3.819,7 Kilometer. ${ }^{26}$ Konzepte hierfür waren schon seit 1924 entwickelt worden. Bereits im August 1932 wurde die Autobahn Köln - Bonn dem Verkehr übergeben. ${ }^{27} \mathrm{Im}$ faschistischen Italien entstanden ebenfalls Autobahnen. ${ }^{28}$ Im Frühjahr 1934 wurde an 15 Baustellen mit dem Bau des neuen Straßentyps Autobahn begonnen. ${ }^{29}$ Die avisierte Zahl von 600.000 Beschäftigten wurde jedoch bei weitem nicht erreicht: Die höchste Zahl der Arbeitsplätze, die im Juli 1936 erreicht wurde, betrug 124.483, hinzu kam etwa dieselbe Zahl in der Zulieferindustrie. ${ }^{30}$ Daher stellt Ritschl fest: „Weder von seinem Umfang her noch gar in seinem zeitlichen Ablauf hat das deutsche Autobahnprogramm irgendwie nennenswert den Wiederaufschwung beeinflussen können." ${ }^{\text {31 }}$ Kurzfristiges Ziel dieser Bauvorhaben war die Beschäftigung möglichst vieler Erwerbsloser und erst langfristig die notwendige Verbesserung der Infrastruktur. ${ }^{32}$ Der Baubeginn an vielen Stellen gleichzeitig diente der propagandistischen Wirkung. ${ }^{33}$

Die inzwischen etwas abgeflaute Debatte in der Forschung, ob die Ankurbelung der Konjunktur, die Aufrüstung oder eine Massenmotorisierung per se der Hauptgesichtspunkt für die nationalsozialistische Verkehrspolitik war, soll hier nicht nachgezeichnet werden. Zumindest für die Zeit bis 1936 ist Henning beizupflichten: „Weder die Belebung der Autoindustrie noch der Autobahnbau geschahen unter rüstungswirtschaftlichen Gesichtspunkten. Vielmehr versuchten beide Maßnahmen, Arbeit zu schaffen und als Initialzündung zu einer allgemeinen Konjunkturbelebung zu wirken. ${ }^{\text {"“34 Besonders }}$

26 Seit 1937 wurde allerdings für den Autobahnbau ein Aufschlag von vier Pfennig auf den Treibstoffpreis erhoben, mehr als ein Zehntel des bisherigen Preises. Erhard Schütz u. Eckhard Gruber: Mythos Reichsautobahn. Bau und Inszenierung der „Straßen des Führers“ 1933-1941, Berlin 1996, S. 142. Dies ist zugleich ein deutliches Zeichen für den Übergang von der Kraftfahrzeug- zur Kraftstoffsteuer. Zatsch (wie Anm. 15), S. 497f.

27 Ebd., S. 461.

28 Kershaw (wie Anm. 25), S. 571.

29 Der erste Spatenstich Hitlers Ende September 1933 hatte nur symbolischen Charakter. Albrecht Ritschl: Hat das Dritte Reich wirklich eine ordentliche Beschäftigungspolitik betrieben? in: Jahrbuch für Wirtschaftsgeschichte 2003, S. 125-140, hier S. 128.

30 Schütz/Gruber (wie Anm. 26), S. 10-12 u. 56f. An diesem Beispiel ist gut die Wirkung und Mythenbildung der NS-Propaganda zu sehen, die die Beseitigung der Massenarbeitslosigkeit mit dem Autobahnbau („Straßen des Führers“) verknüpfte und die bis heute nachwirkt.

31 Ritschl (wie Anm. 29), S. 128. Ähnlich auch Christoph Kopper: Handel und Verkehr im 20. Jahrhundert (= Enzyklopädie Deutscher Geschichte, Bd. 639), München 2002, S. 32.

32 Hansjoachim Henning: Kraftfahrzeugindustrie und Autobahnbau in der Wirtschaftspolitik des Nationalsozialismus 1933 bis 1935, in: Vierteljahrschrift für Sozial- und Wirtschaftsgeschichte 65, 1978, S. 217-242, hier S. 235.

33 Edelmann (wie Anm. 7), S. 177.

34 Henning (wie Anm. 32), S. 241f. Ähnlich auch Kopper (wie Anm. 31), S. 32 und König (wie Anm. 8), S. 152. 
die Unterstützung der Automobilproduktion war wirkungsvoll. Ohne hohes Risiko konnten ,,vorhandene Kapazitäten einer im beginnenden Aufschwung sich befindenden Branche" zur Arbeitbeschaffung genutzt werden. ${ }^{35}$ Die deutsche Automobilindustrie zählte in den folgenden Jahren zu den „Führern der Modernisierung“. Neben der Rationalisierung durch teilweisen Übergang zur Serienproduktion ist eine massive Modernisierung des Maschinenparks erfolgt. ${ }^{36}$

\section{Der DKW-Kleinwagen und der Aufstieg der Auto Union im Nationalsozialismus}

2.1 Der Kleinwagen DKW F-1

Schon Anfang der 1930er Jahre fand das konkrete Vorhaben, einen preiswerten Wagen als „Volksautomobil“ zu schaffen, Niederschlag in den Akten von Vorstand und Aufsichtsrat der Zschopauer Motorenwerke J.S. Rasmussen $\mathrm{AG}$, einer der Vorgängerfirmen der Auto Union $\mathrm{AG}^{37}$ Dies war zu diesem Zeitpunkt sehr erstaunlich und weitsichtig, da die Rahmenbedingungen von der mit voller Wucht einsetzenden Weltwirtschaftskrise, den hohen Betriebsund Unterhaltskosten für ein Automobil bis hin zur mangelhaften Infrastruk$\operatorname{tur}^{38}$ für einen solchen Plan völlig ungenügend schienen. Andererseits stand Sachsen 1925 mit 86 Einwohnern je Kraftfahrzeug zusammen mit Berlin an der Spitze im Reich. ${ }^{39}$ Die Zschopauer Motorenwerke hatten zudem erst 1928 mit dem Modell „P 15“ mit der Produktion von größeren Serien von Personenkraftwagen mit Verbrennungsmotor begonnen. Bisher beschäftigten sie sich schwerpunktmäßig und sehr erfolgreich mit dem Bau von Motorrädern. Der DKW-PKW P 15 wurde mit einem Zweizylinder-Zweitaktmotor mit $600 \mathrm{ccm}$ über die Hinterachse angetrieben und besaß eine Sperrholzkarosserie. Dieser Wagen wurde im Werk Spandau hergestellt und kostete als zweisitziges Cabriolet 19292.545 RM. ${ }^{40}$

Hinter der Bezeichnung Volkswagen verbarg sich hingegen ein bis auf die Sperrholzkarosserie völlig neuartiger PKW-Typ: der DKW Front F-1 mit einem 18 PS leistenden Zweitaktmotor und einem Hubraum von $600 \mathrm{ccm}$. Nicht nur die Absicht, mit diesem Wagen weite, über die bisherigen Zielgruppen hinausgehende Bevölkerungskreise anzusprechen, war das Neue an

35 Henning (wie Anm. 32), S. 234.

36 Vgl. z.B. J. Adam Tooze: „Punktuelle Modernisierung“. Die Akkumulation von Werkzeugmaschinen im „Dritten Reich“, in: Jahrbuch für Wirtschaftsgeschichte 2003, S. 7998, hier S. 94f., Zitat auf S. 94.

37 Sächsisches Staatsarchiv Chemnitz (im folgenden StAC), 31070 Zschopauer Motorenwerke J.S. Rasmussen AG und Vorgänger, Nr. 1 u. 76.

38 Zum Straßenwesen vgl. Flik (wie Anm. 6), S. 62-70. Das sächsische Straßenwesen galt jedoch in den 1920er Jahren als vorbildlich.

39 Zatsch (wie Anm. 15), S. 429.

40 Eine entsprechende Quittung ist abgebildet in: Frank Rönicke: Der P 15 der Berliner Schauspielerin, in: DKW-Nachrichten, 2000, H. 27, S. 14. 


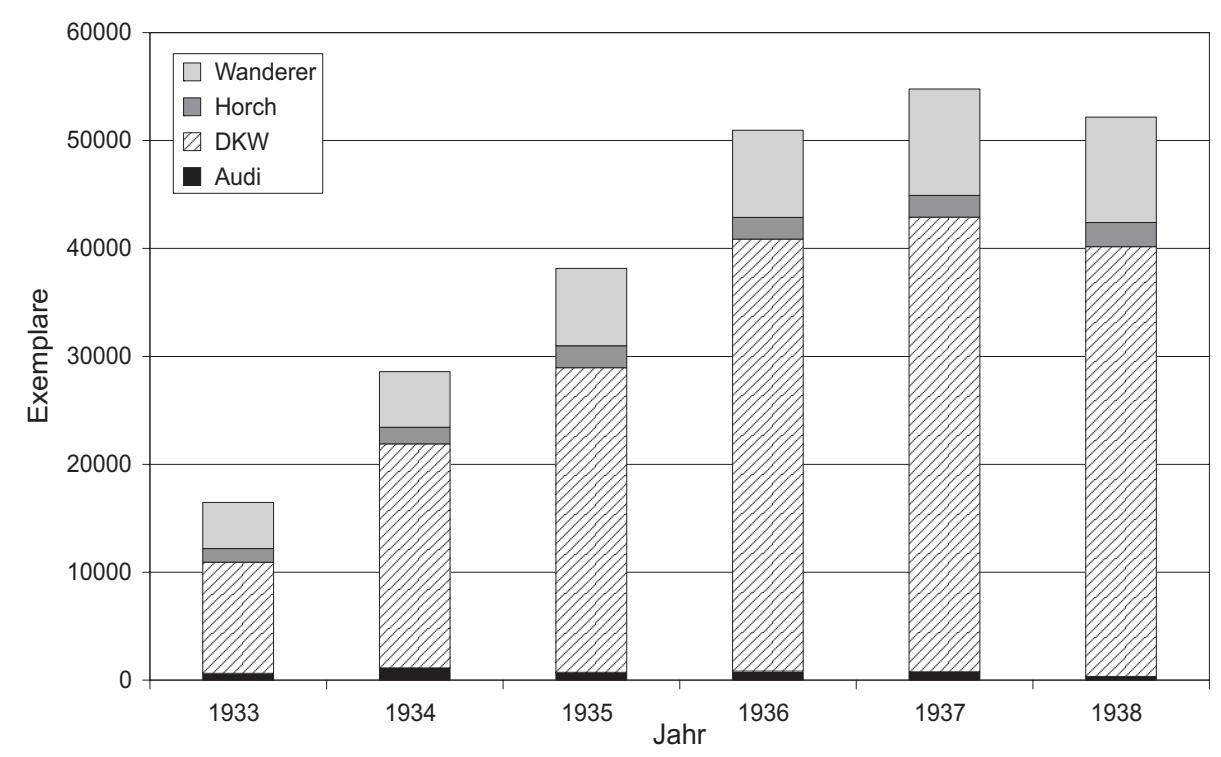

Abb. 1: Der PKW-Absatz der Auto Union AG 1933 bis 1938.

diesem Wagen: Er war zugleich der erste Großserienwagen mit Vorderradantrieb, einzeln aufgehängten Rädern und quer eingebautem Zweitaktmotor! ${ }^{41}$ Dieser innovationsreiche und bahnbrechende Kleinwagen war ein ,groBer Wurf" für DKW. ${ }^{42}$ Die neuen Konstruktionsprinzipien behielten die DKWPersonenwagen bis zur Einstellung der Produktion Mitte der 1960er Jahre bei.

In einer Besprechung zwischen Vertretern der Sächsischen Staatsbank und der Zschopauer Motorenwerke am 15. Januar 1931 bezweifelten die Bevollmächtigten der Staatsbank, „ob der neue Volkswagen alle Voraussetzungen hinsichtlich der Qualität erfüllt“ und in wieweit „,derart neue Fabrikationen wirtschaftlich vertretbar" seien. ${ }^{43}$ Sie sahen sehr deutlich, dass die rapide zu einem umfassenden Konzern ausgebaute Firma schon seit Anfang 1929, also noch vor der Weltwirtschaftskrise, quasi illiquide war und nur durch massive Stützungen der Staatsbank überlebte. ${ }^{44}$ Vor diesem Hintergrund war das Vorhaben, kurz hinter der kostspieligen Einführung der PKWProduktion überhaupt noch einen weiteren, sehr innovativen Wagen heraus-

$41 \mathrm{Zu}$ den mit diesem Wagen zur Serienreife entwickelten Produktinnovationen vgl. zusammenfassend Flik (wie Anm. 6), S. 202.

42 Martin Kukowski: Die Chemnitzer Auto Union AG und die „Demokratisierung“ der Wirtschaft in der Sowjetischen Besatzungszone von 1945-1946 (Beiträge zur Unternehmensgeschichte, hg. von Hans Pohl, Bd. 15), Stuttgart 2003, S. 20.

43 StAC, 31070 Zschopauer Motorenwerke J.S. Rasmussen AG und Vorgänger, Nr. 76, B1. 33.

44 Findbuch, S. XXXVIII u. XXXIX. 
zubringen, ein hohes Risiko. Hinzu kam, dass schon Versuche anderer Firmen, den Frontantrieb mit einzeln aufgehängten Rädern zur Serienreife zu entwickeln, gescheitert waren. ${ }^{45}$ Der neue Wagentyp war auf Initiative des Firmenleiters J.S. Rasmussen von den Audi-Konstrukteuren ${ }^{46}$ Oskar Alt und Walter Haustein sehr kurzfristig entwickelt worden und wurde am 16. Februar 1931 auf der Automobilausstellung in Berlin vorgestellt. ${ }^{47}$ Vorstandsmitglied Dr. Richard Bruhn (1886-1964) erläuterte am 11. März 1931 dem Aufsichtsrat der Zschopauer Motorenwerke in Bezug auf den „Volkswagen (Frontantriebswagen)“, dass „,das bisherige Fabrikationsprogramm durch den Bau dieses Kleinwagens, der zwischen Motorrad und SpandauWagen steht, organisch ergänzt" werde. Er stellte angesichts des Erfolgs auf der Automobilausstellung eine Produktionssteigerung auf über 50 Wagen pro Tag ohne weitere finanzielle Belastung in Aussicht. ${ }^{48}$ Weiterhin sollte versucht werden, eine ,aktive und energische Exportpolitik“ zu betreiben, da das Ausland an dem neuen Typ großes Interesse zeige. ${ }^{49}$ Mit einem Preis von 1.650 RM war der DKW Front F-1 erheblich preiswerter als der DKW P 15 und nur wenig teurer als der billigste deutsche PKW, der P 4 von Opel, der für 1.450 RM angeboten wurde. Die Serienfertigung für das neue DKW-Modell wurde 1931 aufgenommen; 1932 wurden ca. 2.500 Exemplare und 1933 über 8.600 Autos dieses Typs (und teilweise schon des Nachfolgers DKW Front F-2) hergestellt. ${ }^{50}$ Fahrgestellfertigung und Endmontage fanden nach der Bildung der Auto Union im Audi-Werk in Zwickau statt, um die dort vorhandenen Kapazitäten auszulasten. ${ }^{51}$

1933 folgte der DKW Front F-2, nun schon als Modell der Auto Union, dessen billigste Version unter dem Namen „Reichsklasse“ ${ }^{\text {“52 }}$ zum Preis von $1.550 \mathrm{RM}$ angeboten wurde. Bis 1938 wurden bereits über $50.000 \mathrm{DKW}$ Front-Fahrzeuge abgesetzt. ${ }^{53}$ Dennoch darf nicht verkannt werden, dass diese PKW für den „Otto Normalverbraucher“ immer noch unerschwinglich waren. Diese waren immer noch auf das Motorrad angewiesen, dessen unumstrittener Marktführer die Zschopauer Motorenwerke J.S. Rasmussen AG

45 Vgl. allgemein Flik (wie Anm. 6), S. 202f.

46 Die Firma Audi gehörte schon seit 1929 fast vollständig zu den Firmen von J.S. Rasmussen. Findbuch, S. XXVIII.

47 Hans-Rüdiger Etzold, Ewald Rother u. Thomas Erdmann: Im Zeichen der vier Ringe, Bd. 1: 1873-1945, Ingolstadt 1992, S. 235-239.

48 StAC, 31070 Zschopauer Motorenwerke J.S. Rasmussen AG und Vorgänger, Nr. 1, Bl. 13.

49 Ebd., Nr. 76, Bl. 18. Eine mutige Behauptung, weil 1930 der deutsche Außenhandel mit Kraftfahrzeugen erstmals seit 1926 wieder erheblich eingebrochen war. Flik (wie Anm. 6), S. 285f.

50 Peter Kirchberg: Die technisch-konstruktive Entwicklung der Audi-Kraftfahrzeuge von 19091939, Typoskript Dresden 1988 [Expertise für die Volkswagen AG, Wolfsburg], S. 112f.

51 Kukowski (wie Anm. 42), S. 20.

52 Dies war die Bezeichnung für die Standardausführung, die Luxusversion wurde mit „Meisterklasse" bezeichnet.

53 Kukowski (wie Anm. 42), S. 28. 
mit dem Markennamen DKW waren. Die Zielgruppe für die PKW war auf den alten und neuen Mittelstand beschränkt, was zwar eine Vervielfachung der potentiellen Käuferschichten bedeutete, aber mit der Massenmotorisierung, die durch den Volkswagen von den Nationalsozialisten beabsichtigt war, noch wenig gemeinsam hatte.

\subsection{Gründung und Aufstieg des neuen Automobilkonzerns Auto Union ${ }^{54}$}

Die Entwicklung der europäischen Automobilindustrie in der ersten Hälfte des 20. Jahrhunderts ist von Rationalisierungsmaßnahmen und Konzentration geprägt. Sie glich damit der Elektrowirtschaft, die sich seit dem Beginn des 20. Jahrhundert ebenso rasant zu einem bedeutenden Wirtschaftsfaktor entwickelte. ${ }^{55}$ Die Geschichte nicht nur der deutschen Automobilindustrie der 1920er und beginnenden 1930er Jahre ist von geplanten und vollzogenen Fusionen geprägt. Auf Bestreben der Deutschen Bank, die ursprünglich einen umfassenden ,deutschen Autotrust“ schaffen wollte, schlossen sich beispielsweise im Juni 1926 die Automobilfirmen Daimler und Benz zusammen, die schon 1924 eine Interessengemeinschaft eingegangen waren. ${ }^{56}$

1931/1932 entstand aus dem Zusammenschluss der folgenden, aus finanziellen Gründen nicht mehr lebensfähigen Sächsischen Automobilwerke unter Leitung der Sächsischen Staatsbank und mit Unterstützung der Sächsischen Staatsregierung der „Branchenriese“ Auto Union AG:

- Horchwerke AG (1904 in Zwickau von August Horch gegründet),

- Audi-Automobilwerke AG (1909 ebenfalls von August Horch in Zwickau nach seinem Weggang aus der Firma Horch gegründet),

- Zschopauer Motorenwerke J.S. Rasmussen AG (bekannt unter dem Markennamen DKW und seit 1916 im Automobilbau tätig),

- Automobilabteilung der Wanderer AG (sie baute seit 1911/1912 in Chemnitz Automobile, war jedoch schwerpunktmäßig weiterhin ein Fahrradund Maschinenbaubetrieb).

Das ,privatwirtschaftlich geführte Staatsunternehmen“57 war mit anfangs 80 und später über 90\% im Besitz der Sächsischen Staatsbank bzw. des Freistaates Sachsen..$^{58}$ Nach einem verlustreichen ersten Geschäftsjahr 1931/32 stiegen Umsatz und Gewinn stetig und schnell. Die Gründe sind „vielfältige

54 Zur Firmengeschichte bis 1945 siehe zusammenfassend Findbuch, S. XV - C und Kukowski (wie Anm. 42), S. 19-38. Für die Zeit von 1945 bis 1948 vgl. ebd.

55 Die Bedeutung der Automobilindustrie in der ersten Hälfte des 20. Jahrhunderts wird sogar mit der Rolle der Eisenbahn im 19. Jahrhundert verglichen. So schon Davis S. Landes: Der entfesselte Prometheus. Technologischer Wandel und industrielle Entwicklung in Westeuropa von 1750 bis zur Gegenwart, München 1983, S. 406 u. 409.

56 Edelmann (wie Anm. 7), S. 86f.

57 Kukowski (wie Anm. 42), S. 194.

58 Ebd., S. 22. 
unternehmensspezifische und allgemeine wirtschaftspolitische Faktoren ". ${ }^{59}$ Der Konzern verlegte seinen Firmensitz 1936 von Zschopau nach Chemnitz. Er war mit der Marke DKW der größte und bedeutendste deutsche Motorradhersteller und avancierte hinter dem Marktführer Opel zum zweitgrößten Automobilhersteller in Deutschland. Die Marktanteile erreichten 1935/36 den Höchststand mit ca. 35\% bei den Motorrädern und etwa $24 \%$ bei den Personenkraftwagen. Der Anteil der Exporte betrug 1937/38 12\% des Gesamtumsatzes und $27 \%$ des gesamten deutschen Motorrad- und Fahrzeugexports. ${ }^{60}$ Das breitgefächerte Produktionsprogramm von Kleinkrafträdern, Motorrädern, Kleinwagen, Mittelklassewagen, Luxuslimousinen und Stationärmotoren ${ }^{61}$ sowie bedeutende nationale und internationale Rennerfolge ${ }^{62}$ gehörten zu den Faktoren für den stetigen Aufschwung dieses Konzerns. Von seiner Gründung 1932 bis zum Kriegsbeginn 1939 stellte die Auto Union AG etwa 276.000 Personenkraftwagen, 256.000 Motorräder und 20.400 Stationärmotoren her. ${ }^{63}$

\subsection{Die Auto Union und die neue Staatsführung}

Ein Problem für den Konzern war in Bezug auf die neue nationalsozialistische Staatsführung die Vorliebe von Adolf Hitler für Limousinen und Cabriolets der Firma Daimler Benz. Diese Präferenz behielt er seit der Entlassung aus der Haft in Landsberg Ende 1924 bis zu seinem Selbstmord 1945 bei. $^{64}$

Umso wichtiger war es daher für die Leitung der Auto Union, andere Personen der nationalsozialistischen Staats- und Parteiführung für die Marken der Auto Union zu gewinnen und ihnen entsprechend repräsentative Automobile als „Leihwagen“ fast kostenlos zur Verfügung zu stellen. Zuständig für die Verbindung zur NSDAP und damit auch für die Belieferung der politischen Prominenz war zunächst das stellvertretende Vorstands- und NSDAP-Mitglied Klaus-Detlev von Oertzen (1894-1991). ${ }^{65}$ Nach seinem offiziellen Ausscheiden aus der Auto Union AG 1935 wurde der Leiter der zentralen Behördenabteilung Ludwig Heinrich Hensel ${ }^{66}$ damit betraut. Neben

59 Findbuch, S. LXXXIV. Eine zusammenfassende Analyse ebd., S. LXXXIV-LXXXIII.

60 Ebd., S. LXXXIII.

61 Aus dem Produktionsprogramm der Zschopauer Motorenwerke J.S. Rasmussen AG übernommen und weiterentwickelt.

62 Hier ist besonders der bei einem Weltrekordversuch tödlich verunglückte Rennfahrer Bernd Rosemeyer (1909-1938) zu nennen, der von der nationalsozialistischen Propaganda zu einer nationalen Heldengestalt hochstilisiert wurde.

63 Findbuch, S. LXXXIV.

64 Schütz/Gruber (wie Anm. 26), S. 56.

65 Er war 1928-1932 Vorstandsmitglied der Wanderer-Werke. Weil seine Ehefrau nach der nationalsozialistischen Rassenideologie als „nicht-arisch“ galt, wurde er 1935 offiziell entlassen. Er war allerdings weiterhin als freier Mitarbeiter im Export der Auto Union AG für den asiatisch-pazifischen Raum tätig. Nach 1945 war er lange Zeit Management Director Export Division Africa/Australia/Asia des VW-Konzerns. Zu seiner Biografie vgl. Kukowski (wie Anm. 42), S. 41. 
dem NSKK-Führer Adolf Hühnlein (1881-1942), SA-Stabschef Viktor Lutze (1890-1943) und dem Gauleiter von Sachsen Martin Mutschmann (1879-1947?) war die wichtigste Persönlichkeit in diesem Zusammenhang der Preußische Ministerpräsident und der „Zweite Mann“ im Reich, Hermann Göring (18931946). ${ }^{67}$ So erhielt er im September 1935 ein neues Horch-Automobil zur Verfügung gestellt, das durch seinen Chauffeur Klatt im Zwickauer Werk in Empfang genommen werden sollte. Der besonderen Stellung dieses Angestellten wurde aus heutiger Sicht in bescheidenem Umfang Rechnung getragen. In der Anweisung der Behördenabteilung für die Verkaufsabteilung vom 14. September 1935 hieß es klar und eindeutig: „Ich bitte Sie dafür Sorge tragen zu wollen, dass alle Wünsche des Herrn Ministerpräsidenten, die Ihnen durch Klatt übermittelt werden, genauestens erfüllt werden. ... Den Chauffeur Klatt bitte ich bestens zu empfangen; denn er ist beim Ministerpräsidenten eine ungeheure Stütze für unser Fabrikat HORCH. Zumindest vergessen Sie nicht, ihn zu einer guten Zigarre, für die er empfänglich ist, einzuladen. ${ }^{\text {"68 }}$

\section{Die Reaktionen der Auto Union auf den Volkswagen}

3.1 Die deutsche Automobilindustrie und der Volkswagen

Die Förderung der deutschen Automobilindustrie durch die neue Reichsregierung mündete in die Initiative zur Schaffung eines preiswerten Kleinwagens. Dieses Projekt ging Anfang 1934 vom Reichsministerium für Volksaufklärung und Propaganda aus, ehe Adolf Hitler es auf seiner Rede zur Internationalen Automobilausstellung am 7. März 1934 in Berlin vorstellte. Bereits vorher wandte sich das Reichsverkehrsministerium an den „Reichsverband der Automobilindustrie e.V. (RDA)“, und am 12. Februar 1934 kam es zu einer ersten Besprechung. Teilgenommen hatten Vertreter des Reichswirtschafts- und des Reichspropagandaministeriums, Adolf Hühnlein (Führer des NSKK) und der RDA. Die anwesenden Herren der Automobilindustrie wandten sich gegen die Planung eines „Kleinstwagens“ für ca. 1.000 RM und forderten einen Volkswagen mit normalen Abmessungen für bis zu vier Personen. Sie vertraten außerdem die Ansicht, dass eine Senkung der Betriebskosten wichtiger als ein niedriger Anschaffungspreis sei. Auch Hühnlein wandte sich gegen den Plan eines „Volkswagens“, weil er wehrpolitisch völlig uninteressant sei. ${ }^{69}$

Der RDA hielt es jedoch für ratsam, sich der Idee Hitlers nicht vollkommen zu verschließen, um so die Chance zu wahren, dass die deutsche Automobilindustrie den neuen Volkswagen baue. ${ }^{70}$ Die Auto Union verfocht

67 Vgl. Etzold/Rother/Erdmann (wie Anm. 47), S. 377. Sogar Eva Braun besaß einen Horch Achtzylinder, der ihr 1941 von Hitler geschenkt wurde.

68 StAC, 31050 Auto Union AG, Nr. 7823, B1. 1.

69 Ebd., Nr. 866, Bl. 186.

70 Zu den Vorgängen bis 1936 siehe neben Mommsen/Grieger (wie Anm. 17) auch Edelmann (wie Anm. 7), S. 202-207. 
in dieser Zeit zusammen mit den anderen deutschen Automobilherstellern eine agierende und zugleich hinhaltende Strategie, um einerseits das Handeln zu bestimmen und andererseits Zeit zu gewinnen. ${ }^{71}$ Auf einer RDASitzung am 8. Mai 1934 gab Carl Hahn (1894-1961) als Vertreter der Auto Union die Losung aus: „,den Mund zu halten, nach oben zu erklären, dass in Erreichung des Ziels gearbeitet wird, dass dies aber längere Zeit in Anspruch nehmen wird. "'72 Zu diesem Zweck wurde am 22. Juni 1934 ein Konstruktionsvertrag mit dem Ingenieur Ferdinand Porsche (1875-1951) geschlossen, der schon einige Erfahrung in der Konstruktion von kleineren Automobilen gesammelt hatte. Allerdings lagen die zunächst kalkulierten Preise weit über 1.000 RM und widersprachen damit den Vorstellungen der nationalsozialistischen Führung. Ferdinand Porsche stellte am 24. Februar 1936 der deutschen Autoindustrie in einem geheimen Treffen zwei Prototypen eines „Volkswagens“" vor, die nach der Kalkulation in die Nähe der billigsten Wagen von Opel und Auto Union mit ca. 1.400 RM kamen. Der RDA konnte beruhigt konstatieren, dass der Auftrag nicht erfüllt wurde und wohl kaum zu erfüllen sei.

Porsche stellte allerdings auch Hitler im gleichen Monat seine Prototypen vor. Schon fünf Monate später fiel die befürchtete, aber dennoch überraschende Entscheidung: Nach einer weiteren Vorführung von zwei Prototypen am 11. Juli 1936 wurde von der Reichsregierung beschlossen, dass Ferdinand Porsche den Volkswagen weiter konstruieren solle und dass für die Produktion eine völlig neue Anlage und Stadt an der unteren Elbe bei Fallersleben errichtet werden solle. Mit dieser Entscheidung wurde der RDA auf einer internen Sitzung am 27. August 1936 konfrontiert. Einen Tag später schrieb Carl Hahn an Richard Bruhn, dass außerhalb und ohne Rücksichtnahme auf die deutsche Automobilindustrie der Volkswagen kommen werde und er eine ernste Gefahr für das gerade angelaufene Kleinwagengeschäft der Auto Union AG bilde. ${ }^{73}$ Nachdem am 29. Mai 1937 durch die DAF die „Gesellschaft zur Vorbereitung des Volkswagens“ gegründet wurde, begannen am 28. Februar 1938 bei Fallersleben die Bauarbeiten für das neue Werk und die neue Stadt. Die feierliche Grundsteinlegung fand allerdings erst am 26. Mai 1938 statt.

Mit dieser Entscheidung war der RDA und somit die deutsche Automobilindustrie ausgebootet und sah sich plötzlich einem neuen Konkurrenten gegenüber, der von Staat und Partei höchste Unterstützung erhielt. Hiervon waren besonders die beiden führenden in Deutschland produzierenden Kleinwagenhersteller Opel und Auto Union betroffen.

71 So auch König (wie Anm. 8), S. 161.

72 StAC, 31050 Auto Union AG, Nr. 866, B1. 185.

73 Mommsen/Grieger (wie Anm. 17), S. 107. Vgl. auch das Schreiben in StAC, 31050 Auto Union AG, Nr. 866. 
3.2 Volkswagen und Massenmotorisierung - eine Illusion

Der Volkswagen wurde von der „Deutschen Arbeitsfront (DAF)“ mit 50 Millionen Reichsmark gefördert und war vordergründig ein voller Erfolg. Unter der Leitung der „NS-Gemeinschaft Kraft durch Freude (KdF)“ sollte der PKW durch ein spezielles Sparsystem für Bevölkerungskreise erschwinglich sein, die sich bisher keinen PKW leisten konnten. Der „,sozialpolitische Köder“74 des ,Volkswagen-Sparens“ fand in der Bevölkerung nicht den erwarteten Anklang, Ende 1939 gab es 170.000 Sparverträge. ${ }^{75}$ Insgesamt wurden bis Kriegsende 336.000 Besteller bei einer geplanten Jahresproduktion von 500.000 Wagen registriert, von denen 60.000 den Kaufpreis schon komplett angespart hatten. ${ }^{76}$ Der erzwungene Konsumverzicht durch Rationierungen sowie die Ablösesummen für die Abgabe von Fahrzeugen an die Wehrmacht führte zu einer verstärkten Spartätigkeit, wovon das Sparen für den Volkswagen profitierte. ${ }^{77}$

Dennoch war das Sparsystem eine, ,sozialökonomische Utopie“"78 und konnte nicht funktionieren. Die Gesamtkaufsumme belief sich auf 1190 RM (davon 200 RM für eine zwei Jahre laufende Haftpflicht- und Teilkaskoversicherung) plus Überführungskosten. Die Mindestsparrate betrug fünf RM in der Woche. Diesen Betrag konnten große Teile der Bevölkerung nicht aufbringen, weil das Durchschnittseinkommen der Sparer deutlich höher als das eines Industriearbeiters sein musste. Nur etwa ein Drittel der Sparer waren Ersterwerber eines PKW, der Arbeiteranteil lag bei ca. fünf Prozent. ${ }^{79}$ Die in zeitgenössischen Quellen berechneten 69-77 RM für die monatlichen Betriebskosten (davon die Hälfte für Treibstoff) zeigten ganz klar, dass der durchschnittliche Volkswagensparer mit einem Monatseinkommen von 300 bis 360 RM diese Beträge nicht hätte aufbringen können. ${ }^{80}$ Auch die propagierte Nutzung als Freizeitgefährt war Utopie: Etwa 40\% der Fahrzeuge waren als Geschäftsfahrzeuge vorgesehen. Das Geld der Sparer wurde nicht verzinst und allerdings weder für den Werksbau noch für die Rüstung verwendet. ${ }^{81}$

Die Produktion betrug bis Kriegsende nur 630-650 Volkswagen, von denen keiner an einen Volkswagensparer ausgeliefert wurde. Der für diese Fahrzeuge geforderte Preis von 4614 RM (1944) zeigte eindrücklich, dass trotz der geplanten Massenproduktion der offiziell propagierte Preis nur mit sehr starker Subventionierung zu erreichen gewesen wäre. ${ }^{82}$

74 Wolfgang Benz: Geschichte des Dritten Reiches, München 2000, S. 104.

75 Schütz/Gruber (wie Anm. 26), S. 145.

76 Benz (wie Anm. 74), S. 104.

77 Edelmann (wie Anm. 7), S. 217.

78 Mommsen/Grieger (wie Anm. 17), S. 202.

79 Ebd., S. 201 u. König (wie Anm. 8), S. 180.

80 König (wie Anm. 8), S. 186 u. StAC 31050 Auto Union AG, Nr. 7113, Bl. 5. Jerry Sloninger: Die VW-Story, Stuttgart 1981, S. 23, 33 u. 45 schätzt sehr optimistisch, dass immerhin ein Drittel der Sparer sich den Unterhalt eines Wagens hätte leisten können.

81 Mommsen/Grieger (wie Anm. 17), S. 198 u. 200.

82 Ebd., S. 638. 


\subsection{Interne Reaktionen des Konzerns}

Neben den Bemühungen im Rahmen des RDA, den Volkswagen zu verhindern, gab es von der Auto Union die Bestrebungen, dem neuen Kleinwagen eine neue, eigene Produktion entgegenzusetzen. Bereits im Vorfeld der endgültigen Entscheidung für den Volkswagen war die Konzernleitung der Auto Union, die hauptsächlich um den Absatz ihrer Kleinwagen der Marke DKW bangte, höchst beunruhigt. Sie entschied sich für den Versuch, weiterhin das Heft in der Hand zu halten und einen Personenkraftwagen zu entwickeln, der einen qualitativen Vorsprung zum Volkswagen besaß und dennoch recht nahe an dessen Preis vom 990 RM heranrücken sollte. Zu diesem Zweck fand am 3. März 1936, also noch vor der endgültigen Entscheidung der Reichsregierung für den Volkswagenbau, in Zwickau eine streng vertrauliche Sitzung zu ,billigen Wagen“ statt. Der Vorstand war sich mit den Werksdirektoren darüber einig, dass „die Auto Union an dem Problem des Volkswagens nicht vorbeigehen kann" ${ }^{83}$. Da eine drastische Preissenkung des DKW Front Wagens in der Standardausstattung „Reichsklasse“ nicht schnell realisierbar wäre, wurden zwei Aufgaben formuliert: „1.) Das Bestehende muss unter allen Umständen verbilligt werden. Es wird ein Wagen mit RM 1.500.Verkaufspreis verlangt. 2.) Es ist ein völlig neuer Wagen mit RM 1.250.Verkaufspreis zu entwickeln." ${ }^{\text {"84 }}$

$\mathrm{Zu}$ Punkt 1 wurde ausgeführt, dass ca. 380 RM Herstellungskosten einzusparen wären. Die Werksdirektoren konnten aber nur 175 RM mögliche Einsparungen nennen. Die Leiter der Werke Audi, DKW und Spandau wurden beauftragt, weitere Einsparmöglichkeiten zu prüfen. Dieses Ziel wurde anscheinend erreicht, da im Juli 1936 für das DKW Reichsklasse Cabriolet ein Werksabgabepreis von 1.367,05 RM genannt wurde. Mit fünf Prozent Gewinn und 50 RM Vertreterprovision war er für knapp 1.500 RM anzubieten. ${ }^{85}$

Der völlig neue Kleinwagen zu 1.250 RM sollte, wie 1931 der DKW Front F-1, unabhängig von den bisherigen Typen entwickelt werden. Die Planung sah einen Frontantriebswagen mit langer Lebensdauer vor, dessen Innenmaße sich an der DKW-Reichsklasse orientieren sollten. Der Motortyp wurde noch nicht festgelegt, es sollten parallel ein Zwei- und ViertaktMotor mit zwei Zylindern und ca. 20 PS entwickelt werden. Für dieses Projekt wurde ein eigenes Büro mit vorerst vier Konstrukteuren geschaffen. Dieses Vorhaben wurde jedoch nicht realisiert. In welchem Stadium es aufgegeben wurde, ist nicht bekannt. ${ }^{86}$

83 StAC 31050 Auto Union AG, Nr. 3832, Bl. 286.

84 Ebd.

85 Ebd., Nr. 866, Bl. 40

86 Ebd., Nr. 3832, Bl. 287/288. Die Tätigkeit dieses Büros lässt sich in der Aktenüberlieferung der Auto Union nicht dokumentieren. 
Problem und Ziel der Auto Union waren klar erkennbar: Die Gefahr, dass der Volkswagen eine ernsthafte Bedrohung für den Absatz der preiswerten DKW Frontwagen darstellte, wurde klar erkannt. Ebenso war es für die Auto Union nicht möglich, einen Wagen für den geplanten Preis des Volkswagens von 990 RM anzubieten. Es sollte daher versucht werden, für einen etwas höheren Preis zwei qualitativ bessere Wagen als den Volkswagen herauszubringen, um die bisher schon erreichte und noch zu gewinnende Zielgruppe zumindest teilweise nicht an den Volkswagen zu verlieren. In diesem Zusammenhang war die Preisentwicklung in Deutschland für PKW problematisch: Vor allem im Verlauf der Weltwirtschaftskrise sanken die Preise für PKW stark. Diese Entwicklung setzte sich bis 1936/37 fort: 1936 bot die Auto Union ein DKW Front-ZweisitzerCabrio mit 600ccm-Zweizylindermotor an, das mit $1.650 \mathrm{RM}$ zum gleichen Preis angeboten wurde wie 1931 der Front F-1. ${ }^{87}$ Angesichts dieser Entwicklung ergriffen die beiden größten Hersteller von Kleinwagen in Deutschland die Initiative: Um einen ruinösen Preiskampf in Abwehr der Volkswagen-Konzeption zu vermeiden, trafen sich am 29. November 1937 Vertreter der beiden Hersteller Opel ${ }^{88}$ und Auto Union in Frankfurt a.M. Hier wurden detaillierte Preisabsprachen getroffen und vor allem den von offizieller Seite geforderten, weiteren Preissenkungen eine klare Absage erteilt. Außerdem wandte man sich gegen den Vorstoß der Wirtschaftsgruppe Fahrzeugbau, dass keine Automobilfirma, wie z.B. die Auto Union, Fahrzeuge aller Klassen herstellen dürfe. ${ }^{89}$

Die Auto Union beschritt mit den skizzierten Bemühungen um Preissenkungen, Neuentwicklungen und Preisabsprachen einen anderen Weg als die Opel AG. Diese nahm angesichts der Rohstoffkontingentierungen und in Erwartung des Volkswagens ihren erfolgreichen Kleinwagen P 4 nach Produktionseinschränkungen 1937 im Jahr 1938 endgültig vom Markt. ${ }^{90}$ Die Auto Union hatte bei der Beschaffung der Rohstoffe nicht so große Probleme wie Opel, weil die Kleinwagen der Auto Union durch den im Gegensatz zum Viertaktmotor weniger materialintensiven Zweitaktmotor und wegen der Holzkarosserie nicht so stark von rüstungsrelevanten und daher streng bewirtschafteten Materialien abhängig waren. ${ }^{91}$ Zudem galt die Opel AG nicht als deut-

87 Etzold/Rother/Erdmann (wie Anm. 47), S. 292-297.

88 Die Firma Opel, die seit 1899 Kraftfahrzeuge herstellte, hatte bereits 1924 einen erfolgreichen Kleinwagen, den „Laubfrosch“ (offizieller Name 4/12 PS) nach dem Vorbild des 1922 von Citroen entwickelten 5 CV herausgebracht, von dem bis 1931 etwa 120.000 Stück abgesetzt wurden. Flik (wie Anm. 6), S. 146. Bereits am 20.9.1935 war eine ähnliche Vereinbarung zwischen führenden Motorradherstellern in München getroffen worden. Die Preise anderer Firmen wurden erhöht und näherten sich so den Preisen der DKWMotorräder wieder an. StAC, 31050 Auto Union AG, Nr. 837.

89 Ebd., Nr. 5600.

90 König (wie Anm. 8), S. 165.

91 Reinhold Bauer: „Einfach, robust, sparsam, preiswert“. Die DKW-Konzeption als Leitbild für den PKW-Bau der sächsischen Auto Union AG und ihrer ost- wie westdeutschen Nachfolgebetriebe, in: Blätter für Technikgeschichte 62, 2000, S. 143-176, Zitat S. 145. 
sches Unternehmen ${ }^{92}$ und wurde daher nicht in der gleichen Weise wie die deutschen Automobilproduzenten unterstützt. Aus diesen Gründen konnte die Auto Union von der Mitte der 1930er Jahre einsetzenden ,zweiten Motorisierungswelle“ besonders profitieren.

Ein weiteres Problem sah die Konzernspitze in der drohenden Abwanderung von potentiellen Kunden zum neuen, preisgünstigeren PKW. Dies betraf hauptsächlich die Kunden der Marke DKW, die sich natürlich lebhaft für das neue Angebot interessierten. Eine solche Abwanderungstendenz kann im Mai 1939 anhand der Bestellungen für Volkswagen im sächsischen Oppach belegt werden. Dort hatten unter anderen ein Bahnbeamter, ein Zollbeamter und ein Prokurist eine Bestellung für den Volkswagen aufgegeben. „Alles also Leute, die ohne KdF-Wagen bestimmt für DKW in Frage gekommen wären. “93 Das „Volkswagen-Projekt" wurde allerdings auch, wie zum Beispiel 1938 ein Händler aus Greifswald berichtete, von Kunden der Marke Wanderer und damit vom Käuferkreis einer Marke, die preislich eindeutig über den Wagen der Marke DKW angesiedelt waren, stark beachtet. ${ }^{94}$ Aber nicht nur der Verkauf im Inland, sondern auch der Export bekam die neue Konkurrenz zu spüren.

Aus dem Ausland kamen Beschwerden von Vertretungen und Händlern der Auto Union über die massive Werbung für den Volkswagen, die die potentiellen Kunden und auch die Händler selbst verunsicherte. So teilte die argentinische Vertretung Fehling Hnos. der Export-Leitung in Chemnitz am 29. März 1939 mit, „dass die Propaganda für den Volkswagen im Ausland immer größere Formen annimmt. “ Sie fanden ,in den Händen vieler Interessenten bereits Kataloge über den Volkswagen in mehreren Sprachen, die unseren DKW-Verkäufern entgegen gehalten werden. ${ }^{“ 95}$ Solche Beschwerden waren kein Einzelfall. ${ }^{96}$ Die Firmenleitung konnte lediglich immer wieder darauf hinweisen, dass die Produktion des Volkswagens noch nicht angelaufen sei und in den ersten Produktionsjahren wegen der starken Inlandsnachfrage an einen Export nicht zu denken sei.

Am 4. März 1939 konnte die Konzernspitze dieses Thema nach mehreren vergeblichen Anläufen auf einer Besprechung im Reichswirtschaftsministerium (RWIM) zur Sprache bringen. „Es wurden seitens des Herrn Dr. Bruhn die dem RWIM bereits bekannten und öfters geschilderten Bedenken zum Ausdruck gebracht, die gegen die vorzeitige und unsachgemäße Exportpropaganda des KdF-Wagens vorliegen." Es seien inzwischen auch Fälle

92 Mitte März 1929 kaufte der amerikanische Automobilkonzern General Motors Corporation (GMC) 80\% der Opel AG, Edelmann (wie Anm. 7), S. 116f.

93 StAC, 31050 Auto Union AG, Nr. 319, B1. 243.

94 Ebd., B1. 299r.

95 Ebd., Nr. 3676, B1. 86.

96 Weitere Beispiele aus der ganzen Welt mit beiliegenden Presseausschnitten, Flugblättern und Broschüren enthält ebd., Nr. 714. 
bekannt, ,in denen die Volkswagenfabrik durch Vermittlung halboffizieller Stellen an unsere Vertreter herangetreten sei, um sie für den Export des Volkswagens zu interessieren und die notwendigen Auskünfte über die Marktlage in dem betreffenden Land zu erhalten."Als Beispiel wurde Nigeria erwähnt. Ministerialdirigent Dr. Schlotterer stimmte Dr. Bruhn zu, dass Hemmnisse für den DKW-Export nicht entstehen dürften, und sagte zu, im Sinne der Auto Union tätig zu werden. ${ }^{97}$ Der RDA wandte sich ebenfalls mehrfach in dieser Angelegenheit an das Ministerium. In einer streng vertraulichen Besprechung am 5. Mai 1939 mit Vertretern der deutschen Automobilindustrie und der Volkswagenfabrik kam ebenfalls dieses Thema zur Sprache. In dieser Sitzung wurde von Vertretern von Volkswagen offiziell zugesichert, dass vor 1943/44 nicht an einen Export von Volkswagen zu denken sei. ${ }^{98}$ Die Sitzung wie auch viele Schriftstücke aus dieser Zeit beschäftigten sich jedoch hauptsächlich mit der neu aufzubauenden Kundendienstorganisation für den Volkswagen. Hieran hatten die Vertreter der Automobilfirmen großes Interesse, um auf diese Art und Weise doch noch vom Volkswagen profitieren zu können. Sie waren grundsätzlich bereit, die Ausschließlichkeitsklausel gegenüber ihren Vertretungen und Händlern in diesen Fällen nicht anzuwenden. ${ }^{99}$ Nachdem 1938 feststand, dass der Vertrieb des Volkswagens nicht über den Automobilhandel laufen sollte, erklärte sich Volkswagen bereit, bei der Wartung private Werkstätten zu berücksichtigen. ${ }^{100}$

\subsection{Einschätzung des Volkswagen-Projekts durch einen externen Experten}

Neben den geschilderten Aktivitäten in Zusammenarbeit mit dem RDA, der Firma Opel und im eigenen Hause wurde wohl 1938 beschlossen, Stellungnahmen und Analysen zum Volkswagen von einem externen Experten heranzuziehen. Es finden sich in den Akten vier Berichte ${ }^{101}$ des Fahrzeugingenieurs Felix Lauscher ${ }^{102}$ aus Berlin. Sie beschäftigten sich im Auftrag der Auto Union mit den anstehenden wirtschaftlichen und technischen Fragen. Lauscher war außerdem u.a. regelmäßig im Auftrag der Wirtschaftsgruppe

97 Ebd., Nr. 3044, B1. 82/83.

98 Ebd., Nr. 1055.

99 Ebd., Nr. 319, B1. 141-148.

100 König (wie Anm. 8), S. 186.

101 Es hat wohl noch mehr Berichte gegeben: In einem Schreiben von Dr. Bruhn an Lauscher vom 11. Januar 1941 dankte Dr. Bruhn für die Berichte des vergangenen Jahres. StAC, 31050 Auto Union AG, Nr. 852, B1. 69. Zur Überlieferung der Unterlagen der Auto Union AG allgemein siehe Findbuch, S. C-CVI.

102 Lauscher war 1941 für die Leitung eines neu zu gründenden Pressedienstes der deutschen Fahrzeugindustrie im Gespräch bzw. wollte zumindest die Gründung in Zusammenarbeit mit der Industrie betreiben. Vgl. StAC, 31050 Auto Union AG, Nr. 852, B1. 5-28 und 3560. Er wurde von der Auto Union anscheinend häufiger zu Marktuntersuchungen herangezogen, vom 12. Februar 1940 ist von ihm ein ausführlicher Bericht über die Marktverhältnisse in Schweden überliefert. Vgl. ebd., Nr. 1279, B1. 58-62. 
Fahrzeugindustrie tätig. ${ }^{103}$ Neben den Fahreigenschaften und der Ausstattung des Volkswagens sowie den zukünftigen Auswirkungen auf den deutschen und internationalen Automobilmarkt lag ein weiterer Schwerpunkt der Ausführungen von Lauscher in den allgemeinen wirtschaftlichen Auswirkungen der zu erwartenden Massenmotorisierung. Die Berichte, die alle als vertraulich gekennzeichnet waren, geben zumindest indirekt einen detaillierten Einblick in die Einschätzung des Volkswagen-Projekts für einen der führenden Automobilhersteller. Die Konzernspitze schätzte diese Berichte sehr, wie sie explizit betonte. ${ }^{104}$ In seinem ersten überlieferten Bericht vom 30. Mai 1938 berichtete Lauscher von der Grundsteinlegung des neuen Automobilwerkes am 26. Mai bei Fallersleben durch Reichamtsleiter Dr. Bodo Lafferentz. Der Berichterstatter pflichtete nicht nur Lafferentz bei, der den Volkswagen als technisches Wunder charakterisierte, sondern bezeichnete auch die prognostizierte Versicherungsprämie von $1 \mathrm{RM}$ je Woche als ein ,preisliches Wunder“. ${ }^{105}$ Lauschers Schätzungen der Unterhaltungskosten beliefen sich für die ersten fünf Jahre auf ca. 69 RM im Monat, wobei die Kosten in den ersten drei Jahren etwas geringer seien, aber dann durch den Bedarf an neuen Teilen und auftretenden Reparaturen steigen würden.

In seinem nächsten Bericht vom 4. August 1938 charakterisierte Lauscher den Volkwagen als volkswirtschaftliches Energiezentrum, ,das auf allen mit dem Kraftfahrzeug zusammenhängenden Gebieten, kurz in dem gesamten vom Kraftfahrzeug und seinen Auswirkungen erfüllten Raum Erschütterungen auslöst, die durch Anlaufzeit und Liefertempo gemildert werden“. ${ }^{106}$ Die Kräfte, die von diesem Energiezentrum ausgehen, wirken sich auf viele Gebiete aus, und es sei die Aufgabe der ,privatwirtschaftlichen Initiative“ 107 (sprich der Auto Union AG), sich die Auswirkungen rechtzeitig klar zu machen und diese freigesetzten Kräfte im positiven Sinne zu nutzen. Der Autor erkannte auch klar die Schwierigkeit, dass die wenigen bisher bekannten Fakten nur die technische und nicht die wirtschaftliche Seite betrafen. Er erwähnte in diesem $\mathrm{Zu}$ sammenhang auch einen vergleichbaren Markt, nämlich den der Volksempfänger, in dem auch der Plan einer wesentlichen Verbilligung in Verbindung mit einer Massenproduktion zum Erfolg führte. ${ }^{108}$ Parallelen fänden sich „für den wettbewerbsmäßigen Einsatz der Markenfahrzeuge gegenüber dem

103 Ebd., Nr. 852, B1. 66/67.

104 So heißt es in einem Schreiben von Dr. Bruhn an Lauscher vom 11. Januar 1941: „Ich nehme die Gelegenheit gern wahr, um Ihnen am Anfang des neuen Jahres zu sagen, wie gern ich Ihre Berichte im abgelaufenen Jahr gelesen habe und dass ich nur wünschen kann, dass Ihre Berichte weiterhin sich auf solchem Niveau, wie bisher gezeigt, halten mögen." Ebd., Bl. 69. Außerdem sind die Berichte häufig mit Randbemerkungen versehen.

105 Ebd., Nr. 7113, B1. 5.

106 Bericht vom 04. August 1938, ebd., Nr. 319, Bl. 1-4, hier B1. 1

107 Ebd., Bl. 2

108 Ebd., Bl. 4. Zum Volksempfänger VE 301 und zum 1938 eingeführten Deutschen Kleinempfänger (DKE) vgl. König (wie Anm. 8), S. 25-99. Die Zahl der Rundfunkteilnehmer 
Volkswagen ..., aus denen die Notwendigkeit deutlich wird, auch auf dem Kraftfahrzeugmarkt den Volkswagen als Grundlage für die aufstockende Markenfahrzeug-Motorisierung zu nutzen““. ${ }^{109}$

Drei Wochen später, am 27. August, erreichte die Auto Union AG ein weiterer vertraulicher Bericht von Lauscher, der sich mit der Technik des Volkswagens im Vergleich mit anderen PKW beschäftigte. ${ }^{110}$ Das direkte Konkurrenzprodukt der Auto Union, ein DKW Front mit einem 0,7 LiterMotor, kam dabei schlecht weg. Trotz des höheren Preises konnte er weder bei Geschwindigkeit, noch bei Steigfähigkeit und Beschleunigung mit dem Volkswagen gleichziehen. Allerdings musste der Autor einräumen, dass diese Vergleiche nur aufgrund der sparsam veröffentlichten Informationen und nicht anhand von Fahrversuchen unternommen werden konnten. Der Autor plädierte dennoch für weitere Anstrengungen in Entwicklung und Verbesserungen in der Gruppe der Kleinwagen bis ca. zwei Liter Hubraum. Damit sollten gleichzeitig zwei potentielle Kundenkreise erreicht werden; sowohl die derzeitigen Käufer dieser Wagen als auch derjenige Personenkreis, der aus dem Kundenkreis der Volkswagenkäufer nachwachsen und sich mit steigendem Einkommen für ein teureres Fahrzeug interessieren sollte. Hier ging Lauscher von der irrigen Annahme rasch steigender Einkommen weiterer Bevölkerungskreise aus. ${ }^{111} \mathrm{Um}$ die genannten Kaufinteressenten besser zu erreichen, sollte die Typenvielfalt im unteren Preissegment auch durch $\mathrm{Zu}-$ sammenarbeit mit anderen Herstellern beschränkt werden. Auf zwei Punkte sollte dabei das Schwergewicht gelegt werden:

1. Die technischen Daten mussten mindestens um $10 \%$ über denen des Volkswagens liegen. Der Verbrauch durfte dafür auch bis zu 30\% höher sein.

2. Die annehmbare Preisklasse für einen solchen Wagen war ein Preis zwischen 80 und $150 \%$ des Preises des Volkswagens. Das sei nur durch Großserienbau möglich.

Mit diesen Vorschlägen wurde die Strategie des Konzerns, durch Preissenkungen eine zwar etwas teurere, aber hochwertigere Alternative zum Volkswagen anzubieten, bestätigt. Außerdem sei laut Lauscher der Volkswagen der Ausgangspunkt für eine Neuordnung des Automobilmarktes und der Neuentwicklung sämtlicher Typen, da die Auswirkungen des Volkswagens nicht

wurde von 1933 bis 1941 verdreifacht, ebd., S. 83. Adolf Hitler hatte schon bei der Eröffnung der Automobilausstellung 1934 diesen Vergleich gezogen, ebd., S. 157 u. 159.

109 Bericht vom 08. August 1938, StAC, 31050 Auto Union AG, Nr. 319, B1. 358.

110 Bericht vom 26. August 1938, ebd., B1. 325-328.

111 Zur Lohnentwicklung im Dritten Reich siehe z. B. knapp Albrecht Ritschl: Wirtschaftspolitik im Dritten Reich - Ein Überblick, in: Karl Dietrich Bracher, Manfred Funke u. Hans-Adolf Jacobsen(Hg.): Deutschland 1933-1945. Neue Studien zur nationalsozialistischen Herrschaft, Bonn 1992, S. 118-152, hier S. 144. 
nur auf die unteren Preisklassen beschränkt bleiben würden. Eine Vorausschau, die sich nach dem Krieg in großen Teilen bestätigen sollte.

In einem weiteren Bericht beschäftigte sich Lauscher mit den wirtschaftspolitischen Auswirkungen des Volkswagens. Er rechnete hier fälschlicherweise durch eine falsche Einschätzung der nationalsozialistischen Politik mit einer politisch-militärischen Entspannung in Mitteleuropa und somit mit einer Verringerung der Beeinträchtigungen der Volkswirtschaft durch die Belastungen der Aufrüstung (,wehrwirtschaftliche Beanspruchungen"). Am Schluss seiner Ausführungen stellte er wiederum in Verkennung der Entwicklung fest, dass die Voraussetzung für das Volkswagenprojekt eine gestiegene Realkaufkraft sei, die er für die nächsten Jahre erwarte. Diese würde natürlich auch der gesamten Kraftfahrzeugindustrie zugute kommen, wenn es sich nicht nur um eine aufkommensneutrale Umschichtung der finanziellen Mittel handele. ${ }^{12}$ Diese nach der Weltwirtschaftskrise bereits beobachtete Steigerung der Realkaufkraft konnte jedoch durch die Arbeitsmarktpolitik der Nationalsozialisten nicht eintreten, die die Lohnsteigerungen bewusst nicht mit dem Wirtschaftswachstum Schritt halten ließen. ${ }^{113}$

\subsection{DKW und Volkswagen: Der direkte Vergleich 1942}

Die bewusst sparsam gehaltene Informationspolitik in Bezug auf die technischen Details des Volkswagens schürte die Neugier der Automobilindustrie und somit auch Gerüchte. Schlechte Nachrichten über den Volkswagen wurden natürlich von der Konkurrenz und somit auch von der Konzernspitze der Auto Union AG gern gehört. So wurde der Firmenleitung von der zentralen Versuchswerkstatt (ZVW) 1939 mitgeteilt, dass beim Volkswagen sich die Höchstgeschwindigkeit, das Gewicht und der Kraftstoffverbrauch zum Negativen hin entwickelt habe, wodurch sich der Vorsprung des geplanten neuen Kleinwagens DKW F-9114 zumindest in Bezug auf die Höchstgeschwindigkeit erhöhe. ${ }^{115}$ Dieser letzte DKW-Frontwagen mit Dreizylindermotor und Kunststoffkarosserie gelangte wegen des Kriegs nicht zur Serienreife.

Erst am 17. Juni 1942 ergab sich die Möglichkeit, ein Exemplar des Volkswagens von Mitarbeitern der Auto Union eingehend untersuchen zu lassen und zu fahren. Lobend und leicht überrascht wurden die Bedienungsfreundlichkeit, die gute Auskleidung der Stirnwand, die Funktion der Türen einschließlich der Schlösser und die hochwertige Abdichtung hervorgehoben. Negativ wurden die Sitze, die Größe des Kofferraums, die Federung und der Geräuschpegel vermerkt. In einer ausführlichen Aktennotiz an Dr. Bruhn 1942 wurde als Fazit ausdrücklich festgehalten: „Alles in allem macht das

112 Bericht vom 21.09.1938, StAC, 31050 Auto Union AG, Nr. 852, B1. 295-298.

113 Siehe z.B. Benz (wie Anm. 74), S. 104.

114 Sein 3-Zylindermotor mit $800 \mathrm{ccm}$ leistete 28 PS und wurde vom VVB IFA auf der Leipziger Frühjahrsmesse 1948 vorgestellt.

115 Bericht der ZVW vom 20. Juni 1939, StAC, 31050 Auto Union AG, Nr. 319, Bl. 232. 
Fahrzeug nicht einen billigen oder behelfsmäßigen Eindruck, aber es nähert sich stark dem Begriff der ,Fahrmaschine ${ }^{6116}$. Das Mindestmass, was an sogenanntem Fahrkomfort geboten wird, wird jedenfalls vom F-9 bei weitem übertroffen. " ${ }^{117}$ Allerdings sollte der Volkswagen auch zu einem merklich niedrigeren Preis als der weiterhin in Planung befindliche DKW F-9 und dessen Vorgänger angeboten werden. Dass der in der Propaganda angegebene Preis für den Volkswagen allerdings völlig irreal war, wurde bereits nachgewiesen.

Das Volkswagenwerk produzierte zu diesem Zeitpunkt längst nur noch Fahrzeuge für die Wehrmacht. Die Auto Union stellte 1940 die Produktion für zivile Zwecke bis auf Kleinserien von Wanderer- und DKW-PKW für den stark schrumpfenden Export ein. Sie entwickelte sich rasch zu einem „verschachtelten Rüstungskonzern“"118. 1944 erreichte der Konzern schließlich „den Kulminationspunkt seiner „Kriegsexpansion“, bis am 14. April 1945 die Produktion der Auto Union endgültig stillgelegt wurde. ${ }^{119}$

Sie führte die Produktion der zivilen PKW und die Weiterentwicklung der DKW-Frontwagen nach dem Krieg in beiden Teilen Deutschlands fort. Die Innovationen des DKW-Kleinwagens und seine stetige Weiterentwicklung führten zu einem ,soziotechnischen Leitbild ,DKW-Konzeption““, das bis weit in die 1960er Jahre hineinreichte. ${ }^{120}$

\section{Zusammenfassung}

Die Anstrengungen der Auto Union AG, sich durch verschiedene Maßnahmen zum Schutz ihrer Kleinwagenproduktion und deren Absatzes auf die neue Konkurrenz des Volkswagens einzustellen und entsprechend zu handeln, waren alle gescheitert:

- Zunächst gab es Versuche, zusammen mit anderen Automobilherstellern die Entwicklung aktiv in ihrem Sinne zu steuern: Die Bemühungen zusammen mit dem RDA 1934 bis 1936, das Volkswagenprojekt zu stoppen und einen Wagen der deutschen Automobilindustrie als Volkswagen $\mathrm{zu}$ entwickeln und zu produzieren, wurden durch den Alleingang von Ferdinand Porsche, Adolf Hitler seinen Prototypen ohne Beteiligung des RDA vorzustellen, abrupt gestoppt. Hieran konnten auch kleine Erfolge, wie die Preisabsprachen mit der Adam Opel AG über die Kleinwagen-

116 Dieser, hier im übertragenen Sinne gebrauchte Begriff bezeichnete ursprünglich Dreiradpersonenkraftwagen. Sie waren in der Inflationszeit und erneut in der Weltwirtschaftskrise weit verbreitet. Flik (wie Anm. 6), S. 85 und Edelmann (wie Anm. 7), S. 138.

117 StAC, 31050 Auto Union AG, Nr. 319, Bl. 20.

118 Kukowski (wie Anm. 42), S. 36.

119 Ebd.

120 Bauer (wie Anm. 91), S. 174. 
preise 1937 oder auch die 1939 erlangte Zusage, vor 1943/44 den neuen Volkswagen nicht zu exportieren, nichts ändern.

- Noch vor der endgültigen Entscheidung für den Bau des Volkswagens außerhalb der deutschen Automobilindustrie plante die Auto Union, für einen etwas höheren Preis zwei qualitativ bessere Wagen als den Volkswagen herauszubringen, um die bisher schon erreichte und noch zu gewinnende Zielgruppe zumindest teilweise nicht an den zukünftigen Volkswagen zu verlieren. Diese Bemühungen verliefen ergebnislos.

- Die Berichte eines externen Fachmanns, die 1938 angefordert wurden, ergaben keine wesentlichen neuen Impulse für die Unternehmensstrategie. Sie stützten die bisherigen Einschätzungen der Konzernspitze und sahen, allerdings in Verkennung der nationalsozialistischen Planungen, die weitreichenden gesamtwirtschaftlichen Konsequenzen des VolkswagenProjekts voraus. Die illusionäre Preis- und Sparpolitik für den Volkswagen wurde nicht thematisiert.

Die bis nach 1940 in kleinem Maße fortgesetzte PKW-Produktion für den Export und vor allem die stetige Weiterentwicklung des DKW-Kleinwagens bildeten jedoch die Grundlage für die positive Entwicklung der „DKW-Konzeption" nach 1945.

Anschrift des Verfassers: Dr. Lutz Sartor, c/o Sächsisches Staatsarchiv/Hauptstaatsarchiv Dresden, Archivstr. 14, D-01097 Dresden. 